\title{
Value of accelerated peritoneal examination time in pediatric nocturnal intermittent peritoneal dialysis
}

\author{
Mohammed Azar"*, Wee-Song Yeo ${ }^{3}$, Aamir Omair ${ }^{2}$ and Khalid Alfakeeh ${ }^{1}$ \\ ${ }^{1}$ King Saud bin Abdulaziz University for Health Sciences, Nephrology Division, Department of Pediatrics, King Abdulaziz Medical City, MNG-HA, Saudi Arabia \\ ${ }^{2}$ College of Medicine, King Saud bin Abdulaziz University for Health Sciences (KSAU-HS), Riyadh, Saudi Arabia \\ ${ }^{3}$ Shaw-NKF-NUH Children's Kidney Centre, Khoo Teck Puat-National University Children's Medical Institute, National University Health System, Level 12 \\ NUHS Tower Block, 1E Kent Ridge Road, Singapore 119228, Singapore
}

\begin{abstract}
Introduction: In clinical practice, most of the pediatric dialysis patients lack optimal dwell time resulting in suboptimal ultrafiltration (UF) and solute clearance. The appropriate dwell time may be possible to delineate from the Accelerated Peritoneal Examination (APEX) time, derived from a standardized peritoneal equilibration test (PET).

Objectives: This primary objective of the study aims to determine the utility of APEX time for optimal UF in pediatric patients on nocturnal intermittent peritoneal dialysis (NIPD). The secondary objective is to analyze the $\mathrm{Kt} / \mathrm{V}$ and creatinine clearance $\mathrm{L} / \mathrm{week} / 1.73 \mathrm{~m}^{2}(\mathrm{CrCl})$ after approximation of optimal dwell time.

Methodology: The study retrospectively analyzed pediatric peritoneal dialysis patients (age range: 1-14 years), from January 2001 to April 2016 followed up at King Abdul Aziz Medical City, Riyadh, Saudi Arabia, Pediatrics Dialysis Unit. This was a retrospective case series based on chart review. Bivariate descriptive analysis was carried out to report the difference in the ultrafiltration before and after the APEX time calculation.

Results: A total of 15 pediatric patients were enrolled in the study. The mean UF significantly improved after determining the dwell time based on the calculated APEX time (189.4 $\pm 44.7 \mathrm{ml}$ vs $140.5 \pm 47.1 \mathrm{ml}, \mathrm{p}<0.001)$. Additionally, the mean UF remarkably improved in both low/low-average and high/high-average peritoneal transporters $(\mathrm{p}=0.006)$. By analyzing small molecule clearance $(\mathrm{Kt} / \mathrm{V}$, and $\mathrm{CrCl})$ in relation to peritoneal transporters, $\mathrm{CrCl}$ significantly improved in low/low average peritoneal transporters $(\mathrm{p}<0.001)$ whereas, it deteriorated $(\mathrm{p}<0.001)$ in high average peritoneal transporters. On the other side, Kt/V did not vary $(\mathrm{p}=0.93)$ between the peritoneal transporters.
\end{abstract}

Conclusion: APEX time in NIPD can be helpful in maximizing the ultrafiltration and $\mathrm{CrCl}$ especially in patients with low and low-average transporters. The APEX time also provided valuable input in optimizing the UF in high and high average transporters.

\section{Introduction}

Pediatric peritoneal dialysis patients depend on optimal dwell time for adequate UF and clearance. In clinical practice, most of the pediatric dialysis patients lack optimal dwell time and resulting in suboptimal UF. It is possible to delineate the appropriate dwell time from the APEX time as derived from a standardized PET [1-3]. The APEX time is represented by the intersecting point between urea and glucose equilibration curves [3]. It is evidenced that optimal APEX time approximates the actual dwell time for good UF, and moreover, twice the APEX time may lead to good clearances [3-6]. The value of APEX time is well recognized and appreciated in adapted peritoneal dialysis (combination sequences of short and long dwells within one peritoneal dialysis session), However, in NIPD it may be a potential parameter in maximizing the UF. Fischbach et al have demonstrated clearly the effectiveness and determination of APEX time in automated adapted peritoneal dialysis. Furthermore, the benefits of varying between short and long dwell times to balance between UF and clearance has been well described in children [3-7]. In NIPD, however, the dwell time cannot be varied. The approximation of the dwell time utilizing the APEX time may aid in optimizing UF and possibly improve the clearance in low peritoneal transporters.

\section{Objectives of the study}

The study aimed to analyze the value of APEX time in optimizing UF in NIPD.

\section{Secondary objectives}

By approximating the optimal dwell time for ultrafiltration according to the APEX time, the status of clearance namely Kt/V and $\mathrm{CrCl}$ were analyzed after three months.

\section{Methodology and materials}

This is a retrospective case series based on chart review. Peritoneal dialysis details were derived from the digital memory cards

Correspondence to: Mohammed Azar, Department of Pediatrics, Division of Nephrology, King Abdulaziz Medical City, King Abdullah Specialized Children Hospital, Mail Code 1940, Riyadh, P. O. Box 22490, Riyadh 11426, Kingdome of Saudi Arabia , Tel: +966-118011111- Ext- 53524, E-mail: shameemazar@gmail.com

Key words: accelerated peritoneal examination time, peritoneal equilibration test, ultrafiltration, low, high average transporters, creatinine clearance

Received: March 19, 2017; Accepted: April 10, 2017; Published: April 13, 2017 
incorporated in the dialysis machines (Fresenius cycler-sleep safe (V2.2X). Both paper chart review (for patient data) and electronic chart review (for lab data) were assessed. All the recruited patients not underwent PET previously and had NIPD with short cycles (9-11 cycles) using bicarbonate-based physiological solution (1.3\% bicavera, Fresenius Medical Care, Homburg, Germany) for 10 hours. In addition, all patients routinely underwent three monthly peritoneal $\mathrm{Kt} / \mathrm{V}$ and peritoneal $\mathrm{CrCl}$ as there was no significant residual renal function $(<100$ $\mathrm{ml} / \mathrm{m}^{2} /$ day of urine output) noted in all our patients. Historical dwell time used during the dialysis were taken as controls. No patients had day dwell during the dialysis. Mean UF was analyzed over the last 30 days. Additionally, all patients underwent standardized PET and APEX time calculation using a standard graphical chart. Mean UF was further analyzed over the subsequent 30 days. The difference in the outcome of ultrafiltration were analyzed in patients before and after APEX time and its relationship with peritoneal membrane status was also assessed. After 3 months, peritoneal $\mathrm{Kt} / \mathrm{V}$ and $\mathrm{CrCl}$ were performed for all subjects. Exclusion criteria for the study included missing data, history of peritoneal dialysis of less than three months, history of peritonitis within three months and patients with peritoneal membrane failure or features of encapsulated peritoneal sclerosis

\section{Study area/setting}

The study retrospectively analyzed peritoneal dialysis patients aged 1-14 years from January 2001 to April 2016 followed up in King Abdul aziz Medical City, Pediatrics Dialysis Unit, Riyadh, Saudi Arabia.

\section{Data collection methods}

Routine PET performed as per standard protocol, in which dialysate urea, glucose, and creatinine values $(0,2,4$ hours), overnight dialysate sample, blood urea, glucose, and creatinine ( 2 hours) were measured from the lab. All the dialysis details were incorporated into the online system to assess membrane status, $\mathrm{CrCl}$, and $\mathrm{Kt} / \mathrm{V}$. APEX time is calculated from the standard graphical chart. Mean ultrafiltration was analyzed over the 30 days from digital memory card incorporated in dialysis machines. All the information's were entered in the peritoneal examination charts.

\section{Data analysis}

Bivariate descriptive analysis was carried out to report the difference in the ultrafiltration before and after the APEX time calculation. Results were reported in terms of mean and standard deviation. SPSS version 20 used for analysis. A p value $<0.05$ was considered to show a statistically significant difference.

\section{Ethical considerations}

This study was approved by the Institutional Review Board at King Abdullah International Medical Research Centre. A copy of the ethical approval is available for review by the Editor.

\section{Results}

A total of 15 pediatric peritoneal dialysis patients were enrolled in the study. There were $10(67 \%)$ girls and $5(33 \%)$ boys with a female to male ratio of 2:1 (Table 1$)$. Their ages ranged from 1-12 years $(6.3 \pm 3.1$ years). The dwell time used before the APEX time ranged between 40 -55 minutes with a mean of $46.1 \pm 4.6 \mathrm{~min}$ (Table 1 ). The calculated APEX time for these patients ranged between 36- 78 minutes, with a mean of $54.7 \pm 16 \mathrm{~min}$. More than half of the patients $(n=8,53 \%)$ were low/low-average transporters, and remaining patients $(n=7,46 \%)$ were high/high average transporters.
Out of 15 patients enrolled in our study, mean UF significantly improved from $140.5 \pm 47.1$ to $189.4 \pm 44.7$ after incorporating the calculated APEX time into the dwell time (Table 2). Moreover, mean UF remarkably improved more in low/low-average as compared to high/high-average peritoneal transporters (Table 3). The mean (SD) were $n=8,70.4 \pm 27.7$ for low/low-average and $n=7,24.4 \pm 25.8$ for high/ high-average peritoneal transporters. In relation to clearance, $\mathrm{Kt} / \mathrm{V}$ did not change $(\mathrm{p}=0.16)$ before and after APEX time (Table 2) whereas in relation to $\mathrm{CrCl}$, significant improvement $(\mathrm{p}=0.04)$ was noted in our study (Table 2 ). The mean (SD) were $n=15,46.8 \pm 7$. By analyzing small molecule clearance $(\mathrm{Kt} / \mathrm{V}$, and $\mathrm{CrCl})$ in relation to peritoneal transporters, $\mathrm{CrCl}$ significantly improved $(\mathrm{p}<0.001)$ in low/low average peritoneal transporters (Table 3 ). The mean $(\mathrm{SD})$ were $n=8,14.8 \pm$ 6.6 whereas, it is deteriorated $(\mathrm{p}<0.001)$ in high average peritoneal transporters $(n=7$, mean $(\mathrm{SD})-3.3 \pm 1.9)$. On the other side, $\mathrm{Kt} / \mathrm{V}$ did not vary $(\mathrm{p}=0.93)$ between the peritoneal transporters (Table 3 ). The mean (SD) were $\mathrm{n}=8,-0.13 \pm 0.35$, for low/low-average peritoneal transporters and $n=7,-0.14 \pm 0.38$ for high/high-average peritoneal transporters.

\section{Discussion}

Most of the studies in pediatric peritoneal dialysis patients are inconclusive on optimal dwell time for better UF and clearance. The dwell time is principally based on individual peritoneal membrane characteristics [7]. Adult peritoneal dialysis patients, who routinely undergo PET, have their peritoneal dialysis prescription based on peritoneal membrane transport status but in contrast, pediatric peritoneal dialysis patients have practical difficulty in performing standardized PET. Hence, most of pediatric peritoneal dialysis patients

Table 1. Clinical and biochemical profile of APEX time.

\begin{tabular}{|c|c|c|c|}
\hline & Mean \pm sd & Min & Max \\
\hline Age (years) & $6.3 \pm 3.4$ & 1 & 12 \\
\hline Kt/V (before APEX time) & $2.2 \pm 0.4$ & 2 & 3 \\
\hline Kt/V (after APEX time) & $2.1 \pm 0.3$ & 2 & 3 \\
\hline $\begin{array}{c}\text { Dwell Time (Minutes) } \\
\text { Before APEX calculation }\end{array}$ & $46.1 \pm 4.6$ & 40 & 78 \\
\hline $\begin{array}{c}\text { Dwell Time (Minutes) after } \\
\text { APEX calculation }\end{array}$ & $54.7 \pm 16.0$ & 36 & 230 \\
\hline $\begin{array}{c}\text { Mean ultrafiltration before } \\
\text { APEX time }\end{array}$ & $140.5 \pm 47.1$ & 65 & 274 \\
\hline $\begin{array}{c}\text { Mean ultrafiltration after } \\
\text { APEX time }\end{array}$ & $189.4 \pm 44.7$ & 112 & 52 \\
\hline $\begin{array}{c}\text { CrCl L/week/1.73m2 } \\
\text { (before APEX time) }\end{array}$ & $40.5 \pm 7.7$ & 22 & 63 \\
\hline $\begin{array}{c}\text { CrCl L/week/1.73m2 (after } \\
\text { APEX time) }\end{array}$ & $46.8 \pm 7.0$ & 40 & 59 \\
\hline APEX: Accelerated Peritol & Examing & \\
\hline
\end{tabular}

APEX: Accelerated Peritoneal Examination time; Kt/v: measures a change in the concentration of urea; $\mathrm{CrCl}$ : Creatinine clearance; $\mathrm{SD}$ : Standard deviation; Min: Minimum; Max: Maximum.

Table 2. Relationship between mean ultrafiltration, $\mathrm{Kt} / \mathrm{v}$ and $\mathrm{CrCl}$ before and after APEX $(\mathrm{N}=15)$.

\begin{tabular}{|c|c|c|}
\hline & Mean \pm sd & p-value \\
\cline { 1 - 2 } Mean ultrafiltration Pre-intervention & $140.5 \pm 47.1$ & $<0.001$ \\
\cline { 1 - 2 } Mean ultrafiltration Post intervention & $189.4 \pm 44.7$ & \multirow{2}{*}{0.16} \\
\hline $\mathrm{Kt} / \mathrm{V}$ Pre-intervention & $2.2 \pm 0.4$ & \multirow{2}{*}{0.04} \\
\hline $\begin{array}{c}\mathrm{Kt} / \mathrm{V} \mathrm{Post} \mathrm{intervention} \\
\mathrm{CrCl} \mathrm{L} / \text { week} / 1.73 \mathrm{~m}^{2}\end{array}$ & $2.1 \pm 0.3$ & \\
\hline $\begin{array}{c}\mathrm{CrCl} \text { L/week/1.73m } \\
\text { Post intervention }\end{array}$ & $40.5 \pm 7.7$ & \\
\hline
\end{tabular}

$\mathrm{Kt} / \mathrm{v}$ : measures a change in the concentration of urea; APEX: Accelerated Peritoneal Examination time; $\mathrm{CrCl}$ : Creatinine clearance; SD: Standard deviation) 
Table 3. Relationship between mean ultrafiltration, $\mathrm{Kt} / \mathrm{v}$ and $\mathrm{CrCl}$ withlow/low average and high/high average transporters.

\begin{tabular}{|c|c|c|c|c|c|c|c|}
\hline \multirow[b]{3}{*}{$\begin{array}{l}\text { Pre- Post intervention } \\
\text { difference }\end{array}$} & \multirow{2}{*}{\multicolumn{3}{|c|}{$\begin{array}{c}\text { Low / Low Average } \\
(n=8)\end{array}$}} & \multirow{2}{*}{\multicolumn{3}{|c|}{$\begin{array}{c}\text { High / High Average } \\
(n=7)\end{array}$}} & \multirow[b]{3}{*}{ p-value } \\
\hline & & & & & & & \\
\hline & Mean & \pm & sd & Mean & \pm & sd & \\
\hline Mean Ultrafiltration & 70.4 & \pm & 27.7 & 24.4 & \pm & 25.8 & 0.006 \\
\hline $\mathrm{Kt} / \mathrm{V}$ & -0.13 & \pm & 0.35 & -0.14 & \pm & 0.38 & 0.93 \\
\hline $\mathrm{CrCl} \mathrm{L} /$ week $/ 1.73 \mathrm{~m}^{2}$ & 14.8 & \pm & 6.6 & -3.3 & \pm & 1.9 & $<0.001$ \\
\hline
\end{tabular}

$\mathrm{Kt} / \mathrm{v}$ : measures a change in the concentration of urea; $\mathrm{CrCl}$ : Creatinine clearance; SD: Standard deviation.

have their prescription based on an assumption of high transport status [8-12]. Younger children have been described to have high peritoneal membrane transport status as compared to older pediatric populations [8]. But a significant number (8 out of 15) of our patient populations were low/low-average peritoneal transporters, and remaining seven were high/high-average peritoneal transporters. Further, pediatric populations are expected to grow and peritoneal membrane status tends to change over the years. Hence, it is important to do the PET test in children and delineate the appropriate dwell time in order to optimize the peritoneal UF and clearance.

Shortening of dwell time has more pronounced effects on $\mathrm{CrCl}$ than on $\mathrm{Kt} / \mathrm{V}$ because creatinine equilibrium across the peritoneal membrane is slower than that of urea. This is more evident and clinically relevant in low peritoneal membrane transporters. Further, peritoneal membrane slow transporters have sustained osmotic gradient and tend to have better UF and poor small molecule clearance. In contrast, high peritoneal membrane transporters have a rapid osmotic gradient and tend to have better small molecule clearance and poor UF [13-17]. Importantly, osmotic gradient tends to shift only after maximizing the dwell point, hence with optimization of dwell time using APEX time, low/and high peritoneal transporters tend to work efficiently well in relation to UF as compared to $\mathrm{CrCl}$. The above hypothesis, as clearly demonstrated by Fischbach, et al. $[3,4]$ explained the effectiveness and determination of APEX time in automated adapted peritoneal dialysis. But so far up to our knowledge none of the studies have been published in describing the value of APEX time in NIPD. It is prudent to identify the appropriate APEX time and explore the significance of its utilization in terms of clearance and UF in NIPD. In addition, by approximating the optimal dwell time, sodium removal can also be enhanced and potentially improves hypertension and fluid overload [18,19].

This study highlights the change in mean dwell time (based on APEX time) from 45 minutes to 62 minutes, led to significant improvement in the mean UF in patients with low/low-average peritoneal membrane transporters (Table 3). Some other studies have also described that low average peritoneal membrane transporters tend to have efficient UF as compared to other peritoneal membrane transporters [13-15]. In addition, our patients with low/low average peritoneal membrane transporters demonstrated statistical significance in $\mathrm{CrCl}$. Other published studies suggesting that $\mathrm{CrCl}$ may deteriorate in low peritoneal membrane transporters and $\mathrm{Kt} / \mathrm{V}$ may not vary significantly between the peritoneal membrane transporters [13,20-23]. In this study, Kt/V did not vary statistically $(\mathrm{p}=0.93)$ both in low/low average and high/ high average peritoneal membrane transporters and it is possibly due to the subtle difference between the approximate and calculated dwell time especially in high peritoneal membrane transporters. The approximation of APEX time may have played a vital role in improving the UF and $\mathrm{CrCl}$ in low peritoneal membrane transporters noted in our study. On the other side, slow osmotic dissipation in low transporters may have reduced the urea clearance $(\mathrm{Kt} / \mathrm{V})$ but it is not evident in this study as well as other published studies [20,22-25]. Mean UF also improved significantly in the patients with high to high average transporters but it is not so with $\mathrm{CrCl}$ which is significantly deteriorated. But on the contrary, Rocco, et al. describes good small molecule clearance in high peritoneal membrane transporters [20]. Hence from this study, it is possible that by shortening the dwell time, $\mathrm{CrCl}$ is significantly affected and thus the patient with high/high average transporters may need a day dwell with icodextrin or shift to adapted peritoneal dialysis to balance between UF and clearance [3,21-28].

Fischbach, et al. [3-5] identified the importance of the APEX in adapted automated peritoneal dialysis and concluded both short and long cycles are needed to maintain the balance between UF and the clearance. However, in our study incorporation of calculated APEX time into dwell time significantly improved the mean UF in NIPD but did not affect both $\mathrm{Kt} / \mathrm{V}$ and $\mathrm{CrCl}$ in patients with low/low average peritoneal membrane transporters. Larger randomized trials are needed to examine the effectiveness of APEX time and its role in improving the UF in children on NIPD.

\section{Conclusion}

APEX time in NIPD can be useful in maximizing the UF and $\mathrm{CrCl}$ especially in patients with low and low-average transporters. Also, APEX time gives a valuable support to high and high average transporters in optimizing the UF. Limitations of our study are that it is a single center study with a relatively small number of patients.

\section{Acknowledgements}

Authors gratefully acknowledge the assistance of all participants in this study and also all staff members of Nephrology center.

\section{Consent}

Ethical approval for the publication of this article is obtained. A copy of the ethical approval is available for review for the Editor.

\section{Conflict of interest}

The authors declare that they have no competing interests.

\section{Authors' contributions}

- Mohammed Azar: Data analysis, writing and review of the manuscript

- Wee-Song Yeo: Review of the manuscript

- Aamir Omair: Statistical Review.

Khalid Alfakeeh: Review of the manuscript.

\section{References}

1. Fischbach M, Zaloszyc A, Schaefer B, Schmitt CP (2014) Optimising peritonea dialysis prescription for volume control: the importance of varying dwell time and dwell volume. Pediatr Nephrol 29:1321-1327. [Crossref]

2. Fischbach M, Dheu C, Seugé-Dargnies L, Delobbe JF (2007) Adequacy of peritoneal dialysis in children: consider the membrane for optimal prescription. Perit Dial Int 2: S167-170. [Crossref]

3. Fischbach M, Lahlou A, Eyer D, Desprez P, Geisert J (1996) Determination of individual ultrafiltration time (APEX) and purification phosphate time by peritoneal equilibration test: application to individual peritoneal dialysis modality prescription in children. Perit Dial Int 1: S557-560. [Crossref]

4. Fischbach M, Issad B, Dubois V, Taamma R (2011) The beneficial influence on the effectiveness of automated peritoneal dialysis of varying the dwell time (short/long) and fill volume (small/large): a randomized controlled trial. Perit Dial Int 31: 450-458. [Crossref] 
5. Fischbach M, Stefanidis CJ, Watson AR; European Paediatric Peritoneal Dialysis Working Group (2002) Guidelines by an adhoc European committee on adequacy of the pediatric peritoneal dialysis prescription. Nephrol Dial Transplant 17: 380-385. [Crossref]

6. Fischbach M, Terzic J, Menouer S, Haraldsson B (2000) Optimal volume prescription for children on peritoneal dialysis. Perit Dial Int 20: 603-606. [Crossref]

7. Fischbach M, Warady BA (2009) Peritoneal dialysis prescription in children. Bedside principles for optimal practice. Pediatr Nephrol 24: 1633-1642. [Crossref]

8. Schaefer F, Klaus G, Mehls O (1999) Peritoneal transport properties and dialysis dose affect growth and nutritional status in children on chronic peritoneal dialysis. $J \mathrm{Am} \mathrm{Soc}$ Nephrol 8: 1786-1792. [Crossref]

9. Roszkowska-Blaim M, Skrzypczyk P (2014) The clinical significance of peritoneal transport in children during first year of peritoneal dialysis. Pol Merkur Lekarski 37: 212-216. [Crossref]

10. Verrina E, Cappelli V, Perfumo F (2009) Selection of modalities, prescription, and technical issues in children on peritoneal dialysis. Pediatr Nephrol 24: 1453-1464. [Crossref]

11. Fischbach M, Haraldsson B (2001) Dynamic changes of the total pore area available for peritoneal exchange in children. J Am Soc Nephrol 12: 1524-1529. [Crossref]

12. Schmitt CP, Zaloszyc A, Schaefer B, Fischbach M (2011) Peritoneal dialysis tailored to pediatric needs. Int J Nephrol 2011: 940267. [Crossref]

13. Tzamaloukas AH, Murata GH, Piraino B, Rao P, Bernardini J, Malhotra D, et al (1998) Peritoneal urea and creatinine clearances in continuous peritoneal dialysis patients with different types of peritoneal solute transport. Kidney int 13: 1405-1411. [Crossref]

14. Blake P, Burkart JM, Churchill DN, Daugirdas J, Depner T, et al. (1996) Recommended clinical practices for maximizing peritoneal dialysis clearances. Perit Dial Int 16: 448456. [Crossref]

15. Diaz-Buxo JA, Rosenblum S, Zazra J, Crawford T (1997) Further characterization and distribution of peritoneal solute transport rates (PTR). J Am soc Nephrol 8: 282.

16. Burkart JM, Schreiber M, Korbet SM, Churchill DN, Hamburger RJ, et al. (1996) Solute clearance approach to adequacy of peritoneal dialysis. Perit Dial Int 16: 457470. [Crossref]

17. Twardowski ZJ, Nolph KO, Khanna R, Barbara F, Prowant, Leonor P, et al. (1987) Peritoneal equilibration test. Perit Dial 7:138-147.
18. Fischbach M, Schmitt CP, Shroff R, Zaloszyc A, Warady BA (2016) Increasing sodium removal on peritoneal dialysis: applying dialysis mechanics to the peritoneal dialysis prescription. Kidney int 89: 761-766. [Crossref]

19. Akonur A, Guest S, Sloand JA, Leypoldt JK (2013) Automated peritoneal dialysis prescriptions for enhancing sodium and fluid removal: a predictive analysis of optimized, patient-specific dwell times for the day period. Perit Dial Int 33: 646-654. [Crossref]

20. Rocco MV (1996) Body surface area limitations in achieving adequate therapy in peritoneal dialysis patients. Perit Dial Int 16: 617-622. [Crossref]

21. Akcakya M, Oymak O (2013) Does peritoneal membrane transport affect peritonea Clearance of beta 2 microglobulin in peritoneal dialysis patients? Turk Neph dial Trans 22: $101-105$.

22. Wilkie ME, Plant MJ, Edwards L, Brown CB (1997) Icodextrin 7.5\% dialysate solution (glucose polymer) in patients with ultrafiltration failure: extension of CAPD technique survival. Perit Dial Int 17: 84-87. [Crossref]

23. Mujais S, Nolph K, Gokal R, Blake P, Burkart J, et al. (2000) Evaluation and management of ultrafiltration problems in peritoneal dialysis. International society for peritoneal dialysis ad hoc committee on ultrafiltration management in peritoneal dialysis. Perit Dial Int 4: S5-21. [Crossref]

24. Mujais S, Vonesh E (2002) Profiling of peritoneal ultrafiltration. Kidney Int Suppl S1722. [Crossref]

25. Van Biesen W, Heimburger O, Krediet R, Rippe B, La Millia V, Covic A, et al. (2010) Evaluation of peritoneal membrane characteristics: clinical advice for prescription management by the ERBP working group. Nephrol Dial Transplant 25: 2052-2062. [Crossref]

26. Rumpsfeld M, McDonald SP, Purdie DM, Collins J, Johnson DW (2004) Predictors of baseline peritoneal transport status in Australian and New Zealand peritoneal dialysis patients. Am J Kidney Dis 43: 492-501. [Crossref]

27. Coester AM, Smit W, Struijk DG, Krediet RT (2009) Peritoneal function in clinical practice: the importance of follow-up and its measurement in patients Recommendations for patient information and measurement of peritoneal function. Nephrol Dial Transplant 2: 104-110. [Crossref]

28. Voinescu CG, Khanna R, Nolph KD (2002) High peritoneal transport: a blessing or curse? Adv Perit Dial 18: 106-111. [Crossref]

Copyright: (C2017 Azar M. This is an open-access article distributed under the terms of the Creative Commons Attribution License, which permits unrestricted use, distribution, and reproduction in any medium, provided the original author and source are credited. 